\title{
Asymmetry in presupposition projection: The case of conjunction*
}

\author{
Matthew Mandelkern \\ All Souls College, Oxford \\ Jacopo Romoli \\ Ulster University
}

\author{
Jérémy Zehr \\ University of Pennsylvania \\ Florian Schwarz \\ University of Pennsylvania
}

\begin{abstract}
Is the basic mechanism behind presupposition projection fundamentally asymmetric or symmetric? This is a basic question for the theory of presupposition, which also bears on broader issues concerning the source of asymmetries observed in natural language: are these simply rooted in superficial asymmetries of language uselanguage use unfolds in time, which we experience as fundamentally asymmetricor can they be, at least in part, directly referenced in linguistic knowledge and representations? In this paper we aim to make progress on these questions by exploring presupposition projection across conjunction, which has typically been taken as a central piece of evidence that presupposition projection is asymmetric. As a number of authors have recently pointed out, however, whether or not this conclusion is warranted is not clear once we take into account independent issues of redundancy. Building on previous work by Chemla \& Schlenker (2012) and Schwarz (2015), we approach this question experimentally by using an inference task which controls for redundancy and presupposition suspension. We find strong evidence for left-to-right filtering across conjunctions, but no evidence for right-to-left filtering, suggesting that, at least as a default, presupposition projection across conjunction is indeed asymmetric.
\end{abstract}

Keywords: presupposition projection, asymmetry, semantics-pragmatics interface, experimental linguistics

\section{Introduction}

Is the basic mechanism behind presupposition projection fundamentally asymmetric, or does it allow for symmetric effects? That is, when processing presuppositions,

* We gratefully acknowledge audiences in Genoa, at the 30th CUNY, and at SALT 27; RA support from Carissa Redfield and Nikhil Lakhani; valuable discussion with Alexandre Cremers, Gennaro Chierchia, Daniel Rothschild, Philippe Schlenker, and Robert Stalnaker; and financial support from NSF-grant BCS-1349009 to Florian Schwarz. 
can we take into account only material that precedes (either in time, linear order, or hierarchical order) a presupposition trigger, or can we also access material that follows the trigger? This is a basic question for the theory of presupposition, which also bears on broader issues concerning the sources of asymmetries observed in natural language: are these rooted in superficial asymmetries of language uselanguage use unfolds in time, which we experience as fundamentally asymmetric —or can they be directly referenced in linguistic knowledge and representations? ${ }^{1}$

In this paper we aim to make progress on these questions by exploring presupposition projection across conjunction. Projection across conjunction has typically been taken as a central piece of evidence that presupposition projection is asymmetric (i.e. presuppositions can be filtered by material to their left, but not by material to their right). Following recent work, we argue that the evidence, however, is much less clear than is commonly accepted once we take into account independent issues about redundancy (Schlenker 2009; Rothschild 2011; Chemla \& Schlenker 2012). Similarly, intuitions about right-to-left filtering in conjunction are muddied by the possibility of presupposition suspension. The question of the influence of order on projection across conjunction thus remains open. Building on recent work by Chemla \& Schlenker (2012) and Schwarz (2015), we investigate this question by using experimental inference tasks to ascertain whether asymmetries persist once we control for redundancy and suspension. In our results, we find that it does: they provide strong evidence for left-to-right filtering of presuppositions across conjunctions, but no evidence for right-to-left filtering.

\section{Background}

Presuppositions are contents associated with certain lexical items or constructions (triggers) which are typically felt to be background information, and which display characteristic projection behavior. First, the inference that the presupposed content is true is generally licensed even when the trigger is embedded in a variety of entailment-cancelling environments (Karttunen (1973)'s holes), e.g. in a question, the antecedent of a conditional, or under an epistemic modal. Thus the inference from (1a) that Mary used to do yoga is also licensed by the other variants in (1):

a. Mary stopped doing yoga.

b. If Mary stopped doing yoga, then Matthew will interview her for his story.

1 See Schlenker 2008a, 2009; Fox 2008; Rothschild 2011; Chemla \& Schlenker 2012; Mandelkern \& Romoli $2017 \mathrm{~b}$ for discussion. We will use 'left-to-right' or 'right-to-left' to describe the potential order effects; for discussion as to whether these effects should be thought of as being based on linear or hierarchical order see Romoli \& Mandelkern 2016; Chung 2017; Chierchia 2009; Ingason 2016; George 2008; see also the Conclusion section below. 
c. Did Mary stop doing yoga?

d. Mary might have stopped doing yoga.

Second, presuppositions characteristically fail to project - they are filtered, in Karttunen's terminology — when their content is entailed by the trigger's local context (we leave informal the notion of local context here; it can be spelled out in various ways). ${ }^{2}$ Thus none of the variants in (2) presupposes that Mary used to do yoga:

(2) a. If Mary used to do yoga, then she stopped (doing yoga).

b. Mary never used to do yoga, or else she stopped (doing yoga).

c. Mary used to do yoga, and she stopped (doing yoga).

In some cases, this is obvious: e.g. (2a) and (2b) clearly do not license the inference that Mary used to do yoga. In other cases, such as (2c), things are more subtle, since (2c) does license the inference that Mary used to do yoga. But this inference is not itself a presupposition. We can see this by embedding (2c), once again, in a presupposition hole, as in (3). In this case we can no longer infer that Mary used to do yoga, showing that this is not a presupposition of the antecedent, (2c).

(3) If Mary used to do yoga and she stopped, then Matthew will interview her for his story.

What the examples above clearly show is that material to the left of a given presupposition trigger is taken into account in filtering its presupposition. The question we are concerned with in this paper is whether the material to the right can also be taken into account. We focus here on conjunctions, so our question is: can right conjuncts play a role in filtering the presuppositions of left conjuncts, or only vice versa? The standard assumption in the literature has been that filtering across conjunction is exclusively left-to-right, based on contrasts like that between (4a) (which has a presupposition trigger in a right conjunct, with the presupposition filtered by the left conjunct) versus (4b) (which reverses this order):

a. Mary used to do yoga and she stopped doing yoga.

b. \# Mary stopped doing yoga and she used to do yoga.

But, as discussed by Chemla \& Schlenker (2012); Rothschild (2008, 2011) a.o., it is not entirely clear what such contrasts show. (4b) is certainly marked, and one explanation for this could be that presuppositions must be filtered by material

2 See Karttunen 1974; Stalnaker 1974; Heim 1983 for classic treatments, and Schlenker 2009 for important recent work. For accounts not based on the notion of local contexts see Schlenker 2008b; Fox 2008; Chemla 2008; George 2008 among others. 
which appears to the left of their trigger, as in (4a). The presupposition in (4b) would consequently project and be the source of the sentence's markedness. But this explanation does not suffice, since presupposition triggers can felicitously appear on their own, in which case the presupposition can be accommodated, as in (5) asserted out of the blue:

Mary stopped doing yoga.

The infelicity of (4b), and its contrast with (4a), thus must (at least in part) have an explanation in terms of independent factors. And, indeed, an independent explanation is ready at hand. (4b) has the structure ${ }^{r} S_{p}$ and $\left.p\right\urcorner$ (where $S_{p}$ is a sentence which presupposes $p$ ). There is something marked about roughly parallel cases that don't involve presuppositions of the form ${ }^{\ulcorner} p^{+}$and $p^{\urcorner}$(where $p^{+}$is a sentence which entails, but is not entailed by, $p$ ), as we can see in the contrast between (6a) and (6b):

a. John is a college student, and he is majoring in English.

b. \# John is majoring in English, and he is a college student.

Most theories of redundancy (following Stalnaker 1978) capture this contrast based on the idea that we generally want our contributions to conversation to augment the common information as they proceed. ${ }^{3}$ Sentences of the form ${ }^{r} p$ and $p^{+\urcorner}$intuitively do this (in a null context); sentences of the form ${ }^{\ulcorner} p^{+}$and $p^{\urcorner}$intuitively do not. Assuming that $S_{p}$ entails $p$-or that the constraint invoked here otherwise takes into consideration presupposed content in some manner-the same explanation extends to cases of the form $\left\ulcorner S_{p}\right.$ and $\left.p\right\urcorner$, such as our (4b) above. Thus it is not clear that the relative infelicity of (4b) as compared with (4a) tells us anything about presupposition projection, as opposed to something more general about redundancy.

To circumvent issues about redundancy, we can modify our test paradigm slightly, following Rothschild 2008; Chemla \& Schlenker 2012. So far, we have contrasted sentences of the form ${ }^{\ulcorner} p$ and $S_{p}{ }^{\urcorner}$versus ${ }^{\ulcorner} S_{p}$ and $\left.p\right\urcorner$, where $S_{p}$ is a sentence which presupposes $p$. Instead, however, we can contrast sentences of the form ${ }^{r} p^{+}$and $S_{p}{ }^{7}$ with sentences of the form ${ }^{\ulcorner} S_{p}$ and $\left.p^{+}\right\urcorner$, as in (8):

(7) a. Mary used to do Jivamukti yoga and she stopped doing yoga.

b. Mary stopped doing yoga and she used to do Jivamukti yoga.

This paradigm circumvents issues about redundancy, because $p^{+}$adds something beyond $S_{p}$ (and likewise $S_{p}$ adds something beyond $p^{+}$); thus, on the basis of issues about redundancy alone, there should be nothing wrong with conjunctions of $p^{+}$and

3 E.g. those in Schlenker 2008a, 2009; theories based on structural simplifications instead predict that both variants will be felt to be redundant; see Katzir \& Singh 2013; Mayr \& Romoli 2016. 
$S_{p}$ in either order. And indeed, strikingly, the contrast in felicity observed in (4a) versus (4b) above seems to disappear in (7), suggesting that that contrast really was about redundancy, and not presupposition projection.

Does this show, then, that presupposition projection across conjunction is in fact symmetric? Not at all. It simply controls for an irrelevant issue concerning felicity. The fact that the result is felicitous could be due to symmetric filtering. But it could also result from felicitous accommodation of the presupposition. To determine whether presupposition projection across conjunctions is symmetric, we need to push further by testing whether presuppositions project equally out of constructions with the form of (7a) and (7b). This question cannot be answerd by looking at simple conjunctions, since the presupposition will be licensed as an inference for both orders, given the logical nature of conjunction and the potential of accommodation. Instead, we need to embed these sentences further under presupposition holes, e.g. in the antecedents of conditionals, and see what projects:

(8) a. If Mary used to do Jivamukti yoga and she stopped doing yoga, then Matthew will interview her for his story.

b. If Mary stopped doing yoga and she used to do Jivamukti yoga, then Matthew will interview her for his story.

The crucial question now is whether (8a) and (8b) license the inference that Mary used to do yoga to the same degree. In evaluating this, there is a final confound which we must be careful to control for: namely, the possibility of presupposition suspension, or local accommodation. Presuppositions sometimes fail to project; e.g. (9) has a coherent, if slightly marked, reading:

(9) Mary didn't stop doing yoga—she never did yoga to begin with!

Local accommodation is a process in which presuppositions triggered in entailmentcanceling environments which generally exhibit projection, like negation and antecedents of conditionals, can nonetheless be suspended. ${ }^{4}$ It is thus crucial in addressing questions of filtering to distinguish failure to project due to local accommodation from failure to project due to filtering, either left-to-right or right-to-left.

The question, then, is whether, after controlling for issues of redundancy-by strengthening the non-presuppositional conjunct-and for local accommodation, we find a difference in levels of projection out of the antecedents of conditionals of the form 'If $p^{+}$and $S_{p}$, then $\mathrm{q}^{\urcorner}$(like (8a)) and 'If $S_{p}$ and $p^{+}$, then $\mathrm{q}^{\urcorner}$(like (8b)), respectively. If so, this would be in line with an asymmetric story; if not, it would suggest a symmetric approach. One option at this juncture is to consult our own intuitions, and there is some precedent for this in the literature; Rothschild (2008,

4 We remain non-committal with respect to theoretical approaches; see Heim 1983 for a classic account. 
2011), for instance, reports that in his judgment, there is no projection from either sentences of the form (8a) or those of the form (8b). But intuitions here are quite subtle- too subtle, it seems to us, to settle without experimental inquiry. In light of this, we conducted two experiments to ascertain speaker judgments about what inferences are licensed by conditionals with these forms.

\section{Experiment 1}

\subsection{Design}

The first experiment employed a simple inference task: participants read a sentence and were asked whether they would draw a given inference from that sentence. In the critical trials, the sentence had the form of a conditional whose antecedent contained a conjunction. We manipulated whether a presupposition trigger appeared in the left or in the right conjunct of that conjunction. (10) illustrates how these elements were phrased and displayed. Participants had to indicate whether they would conclude that the proposition introduced by the presupposition trigger (here, that Mary used to do yoga) holds by pressing ' $\mathrm{F}$ ' or ' $\mathrm{J}$ '.

(10) a. If Mary stopped doing yoga and she used to do Jivamukti yoga, then Matthew will interview her for his story.

b. This leads me to conclude

c. Mary used to do yoga.

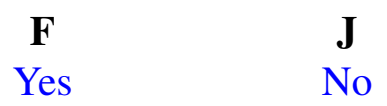

The rationale is that if the presupposition introduced by the trigger projects, participants should reason that their (fictional) interlocutor took its truth for granted, and correspondingly endorse the inference that it holds. On the other hand, if it fails to project (due to filtering or local accommodation), its truth should be taken as hypothetical since the presupposition would only contribute to the scope of the conditional, rendering the inference as unwarranted. By manipulating conjunct order (Conj-Ps-First as in (8a) vs. Conj-Ps-Second as in (8b)), we tested for (a-)symmetry in projection. If only preceding material can filter the presupposition of a given trigger, filtering is only available when the trigger appears in the second conjunct. Correspondingly, finding projection to be less present in such trials (as reflected in the rate of inference endorsements in our task), compared to trigger-first trials, would provide evidence for asymmetry in projection. In contrast, if conjunct order does 
not affect the presence of projection, this would indicate projection to be symmetric, with both left-to-right and right-to-left filtering as equally available options.

Given that in addition to filtering, lack of projection can also be due to local accommodation, absolute inference rates are not enough to establish the presence or absence of filtering. To control for this, our design also included variants of test items as in (11), with a non-conjunctive presuppositional antecedent (Simple-Ps).

(11) If Mary stopped doing yoga, then Matthew will interview her for his story.

As there is no reason to assume that the availability of local accommodation is affected by the particular internal structure of the antecedent, this provides a baseline for projection within the task. To the extent that the critical variants with conjunctive antecedents allow for an additional mechanism to prevent projection, namely filtering, this should be reflected in relatively lower inference endorsement rates than in the simple control variants, which only allow for local accommodation.

At the other extreme, we also included a baseline for non-projection in the form of conditionals with simple non-presuppositional (Simple-No-Ps) antecedents like (12). This establishes how likely participants were to infer that content introduced in the antecedent would hold, even in the absence of presuppositional expressions.

(12) If Mary used to do yoga, then Matthew will interview her for his story.

Theoretically speaking, as far as we are aware, all accounts that predict asymmetry in filtering (as well as several symmetric accounts) also predict that filtering always takes places when the filtering material precedes the trigger in a conjunction. This predicts that there will be no difference in inference rates between Conj-PsSecond and Simple-No-Ps.

\subsection{Materials, Procedure, and Participants}

We created 32 critical items which came in four variants corresponding to the four conditions described above. (13) provides a schematic illustration. The question associated with these items was always whether participants inferred $p$.
a. If $S_{p}$ and $p^{+}$, then $q$.
b. If $p^{+}$and $S_{p}$, then $q$.
c. If $S_{p}$, then $q$.
d. If $p$, then $q$.

Conj-Ps-First

Conj-Ps-Second

Simple-Ps

Simple-No-Ps

Four counter-balanced lists of items were created, so that participants saw eight repetitions in each of these four conditions, while only seeing each item in one 
version. We used four different presupposition triggers to create the items: aware (that), happy (that), stop, and continue. As a result, each participant saw two different trials in each condition per trigger. We also included 48 filler items. 12 of them were structurally identical to (13a) and (13b) but asked participants whether they inferred $p^{+}$or $q$ (rather than $p$ ); 12 were structurally identical to (13c) but asked participants whether they inferred $q .16$ filler items were structurally parallel to (13a) and (13b) but had non-presuppositional embedding expressions (hope (that), doubt (that), try (to), plan (to)) and asked participants whether they inferred $p, p^{+}$or $q$. Finally, 8 filler items consisted of non-conditional, conjunctive sentences of various forms and asked participants whether they inferred one of the conjuncts.

Participants started the experiment with two practice trials where the separate elements in the display were revealed step by step, with tool-tips explaining the nature of the task: they were invited to imagine that a given sentence (corresponding to (10a)) was produced by a fictional interlocutor and to indicate whether they would conclude that a relevant statement $(\approx(10 \mathrm{c}))$ held based on what their interlocutor said. The practice trials were designed as clear cases of endorsement and non-endorsement of the conclusion (using (non-)entailments of an unembedded conjunction and disjunction respectively), and feedback on their responses provided through tool-tips reinforced the notion that the fictional interlocutor should be deemed reliable. An archived version of the experiment can be accessed online at http://spellout.net/ ibexexps/SchwarzLabArchive/IncrSymExp1/experiment.html?Home=T.

We recruited 50 native English speakers online from the participant pool of Prolific.ac. They were rewarded $£ 1.80$ for their participation and took an average of 16 minutes to complete the task. They were distributed in four groups, following a latin-square distribution of the four conditions over the 32 items, so that each item appeared in each condition across participants in the data we collected.

\subsection{Results}

The graph in Figure 1 shows the mean inference rates in each of the four critical conditions. Given that accuracy on fillers was close to ceiling throughout, we didn't remove any data point from our analyses. We conducted analyses using mixed-effect logistic regression models (lme 4 version $1.1-13 ; R$ version 3.3.3) predicting the observation of a 'Yes' answer as a function of Condition (Conj-Ps-First vs. Conj$P s-S e c o n d$ vs. Simple-Ps vs. Simple-No-Ps). The models included random intercepts for participants and items (but no random slopes, due to failures to converge). We found significant differences between every pair of conditions (all $p$ 's $<0.01$ ) except between Conj-Ps-First and Simple-Ps $(p=0.315)$. 


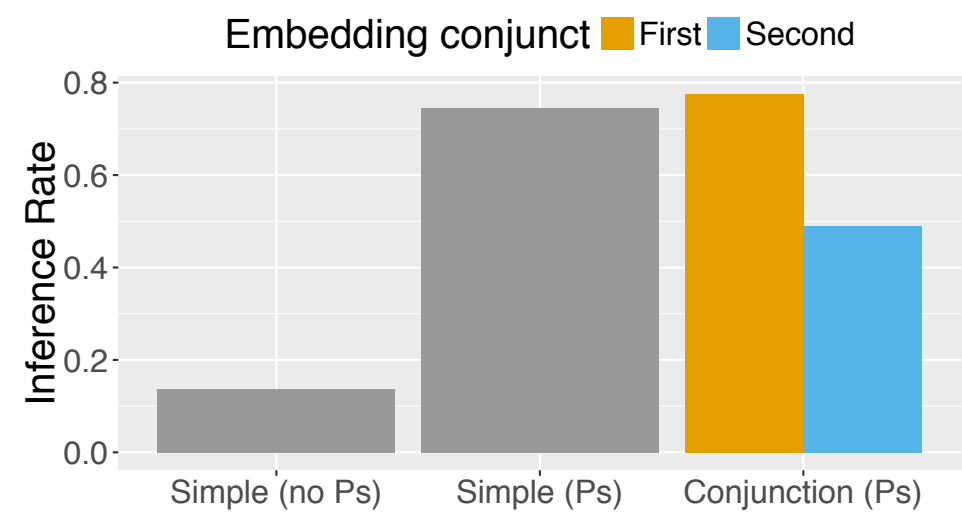

Figure 1 Mean inference rates of $p$ per condition in Experiment 1

\subsection{Discussion}

With regards to our central question of (a-)symmetry, we found a significant effect of conjunct order on inference endorsement rates, with higher rates in Conj-PSFirst than in Conj-Ps-Second. This is consistent with an asymmetric projection mechanism, as the availability of left-to-right filtering should lead to fewer inference endorsements in Conj-Ps-Second. Moreover, the lack of a significant difference between Conj-Ps-First and the projection baseline provided by Simple-Ps indicates that there is no effect of right-to-left filtering in our task. This suggests that filtering is more available in Conj-Ps-Second, and possibly not available at all in Conj-Ps-First, in line with an asymmetric projection mechanism.

A rather surprising aspect of our results is that while the inference endorsement rates for Conj-Ps-Second were significantly lower than for Conj-Ps-First, they also were significantly higher than in the non-projection baseline control provided by Simple-No-Ps. Given that the standard theoretical assumption is that filtering, when available, always takes place, finding any amount of projection judgments in a configuration where filtering is clearly available is unexpected. While we are not in a position to fully explain this aspect of our data here, we note that there are potential factors that could bear on this independently of the nature of the projection mechanism. The most obvious possibility is that participants failed to perceive the entailment relationship between the non-presuppositional conjunct and the presupposition. ${ }^{5}$ In that case, filtering would not be expected regardless of order. Thus, this surprising finding may be compatible with standard assumptions.

5 The manipulations in our materials to create the entailment relationship were varied, and included the use of lexical hyponomy (coffee vs. espresso), compounds (toys vs. toy cars), and world knowledge (going to France vs. going to Paris). 
Comparing observed projection with the degree to which subjects explicitly endorse the entailment in question may indicate whether this is the correct explanation; further exploration is left for future work.

Returning to our main interest, while our results are consistent with asymmetric accounts of projection, there could be alternative explanations of the inference endorsement contrast based on conjunct order. First, two of the triggers employed (aware and happy) were in principle open to syntactic ambiguity, so that the second conjunct in the Conj-Ps-First versions could in principle be interpreted in the scope of the trigger (they could be interpreted as ${ }^{\ulcorner} \ldots$ aware/happy that $\left[p \text { and } p^{+}\right]^{\urcorner}$, leading to projection of $p^{+}$). However, this interpretation seems dispreferred; e.g. 'If Douglas is happy that Sharon is moving to Africa and she is moving to Kenya, then he will help her with the move' doesn't seem to have a natural interpretation on which Douglas is happy that Sharon is moving to Kenya. Second, if this had driven the asymmetry, the effect of order should not have been present for the other two triggers (stop and continue). But the asymmetry is present across triggers, so this worry is not substantiated by our data.

A second concern, however, is more serious. The format of the critical versions of our sentences differs from the controls in that only the former had conjunctions in the antecedent of the conditional, which leaves room for independent effects based on conjunction that are unrelated to the presuppositions at play. For example, the second conjunct could have received a parenthetical interpretation, as illustrated in (14).

(14) a. If Mary stopped doing yoga — and she used to do Jivamukti yoga—, then Matthew will interview her for his story.

b. If Mary used to do Jivamukti yoga — and she stopped doing yoga—, then Matthew will interview her for his story.

(14a) sounds like a plausible utterance to us: parentheticals introduce information as backgrounded, and the parenthetical conjunct $\left(p^{+}\right)$in (14a) can naturally be interpreted as adding background details (outside of the conditional) to the information in the antecedent, details that naturally can be seen as reinforcing the relevance of the conditional. In contrast, the parenthetical conjunct in (14b) $\left(S_{p}\right.$, which doesn't entail $p^{+}$) does not lend itself to being seen as adding details to the first conjunct in a parallel manner. Likely due to the lack of such an interpretation, this sentence seems less felicitous to us under a parenthetical construal. Moreover, it's clear that no parenthetical interpretation of the first conjunct of the antecedent is possible. So a parenthetical construal is available in our Conj-Ps-First sentences. And this construal would favor endorsing the inference that $p$ holds. Given the lack of availability of such a construal for the Conj-Ps-Second sentences, this could be responsible for the contrast in inference endorsement rates based on conjunct order in our data. If this 
were so, then this contrast would not necessarily show that there is an asymmetry, with left-to-right filtering being more available than right-to-left filtering; the apparent contrast could rather be the result of at least some inference endorsements in the Conj-Ps-First condition being due to parenthetical interpretations. This worry is further corroborated by an inspection of the filler items using conjunctions with non-presuppositional embedding verbs. While these were not rigorously controlled in relevant ways, and the data was more limited in that only some of these asked whether $p$ (as opposed to $p^{+}$or $q$ ) could be inferred, they yielded at least suggestive evidence that there could be some asymmetries in endorsements of $p$ even in the absence of a presupposition trigger.

In order to control for a potential alternative explanation in terms independent of the properties of presupposition projection, we conducted a second experiment. The key change was for our critical items to include control variations where the presuppositional expressions were replaced by non-presuppositional ones, while leaving the conjunctions untouched otherwise.

\section{Experiment 2}

\subsection{Design}

The design was parallel to Expt. 1, except that the control variations of the critical items now comprised versions where the presuppositional verbs were replaced by non-presuppositional ones, yielding non-presuppositional sentences with either conjunct order Conj-No-Ps-First ( ${ }^{\text {If }} S_{(p)}$ and $p^{+}$, then $\left.q\right\urcorner$, where $S_{(p)}$ is a sentence which embeds but does not presuppose $p$ ) and Conj-No-Ps-Second ( ${ }^{\circ}$ If $p^{+}$and $S_{(p)}$, then $q^{\urcorner}$). This manipulation allowed us to control for potential conjunct-order effects independent of the presuppositional component, such as the one considered above. Since the new control items didn't contain presupposition triggers, the inference rates observed in these conditions are reflective of what happens in absence of any filtering strategy. Thus, if there are general order effects present in both presuppositional and non-presuppositional sentences, any additional presupposition-based asymmetries should be reflected in even greater order effects for the presuppositional variants. In other words, the hypothesis that there is a preference for left-to-right over rightto-left filtering predicts an interaction between Embedding type ( $P s$ vs. No-Ps) and Order (First vs. Second).

\subsection{Materials and Participants}

The materials from Expt. 1 were adapted slightly to maximize plausibility across conditions. Presupposition triggers were paired with non-presuppositional expressions 
as follows to maximize plausibility in all variants while leaving the remainder of the sentences unchanged: happy (that)/hope (that), aware (that)/sure (that), stop/frown on ( $V$-ing), continuelenjoy ( $V$-ing). (15) lists the resulting 4 conditions. (16) illustrates the Conj-No-Ps-Second version of our running example (8). The Simple-Ps and Simple-No-Ps baseline conditions were included as (between-item) fillers.
a. If $S_{p}$ and $p^{+}$, then $q$.
b. If $p^{+}$and $S_{p}$, then $q$.
c. If $S_{(p)}$ and $p^{+}$, then $q$.
d. If $p^{+}$and $S_{(p)}$, then $q$.

Conj-Ps-First

Conj-Ps-Second

Conj-No-Ps-First

Conj-No-Ps-Second

(16) If Mary used to do Jivamukti yoga and she frowns on doing yoga, then Matthew will interview her for his story.

As before, 32 critical items in four versions were counter-balanced across four lists. The baseline conditions Simple-Ps and Simple-No-Ps were varied within 16 filler items, so that each participant saw eight repetitions of each. Thirty-two additional fillers were re-used from Expt. 1. An archived version of the experiment can be accessed online at http://spellout.net/ibexexps/SchwarzLabArchive/IncrSymExp2/ experiment.html? Home $=\mathrm{T}$.

We recruited 49 students (all self-reported native speakers of English) through the Psychology Subject Pool at the University of Pennsylvania, who took the experiment on a lab computer and received course credit for their participation. ${ }^{6}$

\subsection{Results}

The graph in Figure 2 shows the mean inference rates in the baseline (grey) and critical (orange and blue) conditions. Accuracy was high throughout so no data were removed before analysis. The same statistical analyses as reported for Expt. 1 were conducted. Models predicting responses from the four critical conditions included two predictors, Embedding Type (Ps vs. No-Ps) and Order (First vs. Second) and their interaction, as well as random intercepts for participants and items (adding random slopes led to high random effect correlations, but did not alter the pattern of statistical outcomes). There were significant simple effects of both predictors (at all levels) as well as a significant main effect for Embedding Type and Order, and a significant interaction (all $p$ 's $<0.05$ ). Models predicting responses from the test and baseline conditions (between-item) included one four-leveled predictor (Conj-Ps-First vs. Conj-Ps-Second vs. Simple-Ps vs. Simple-No-Ps), a random slope

6 Note that the replication of the results from Expt. 1, detailed below, thus validates the paradigm across two populations. 


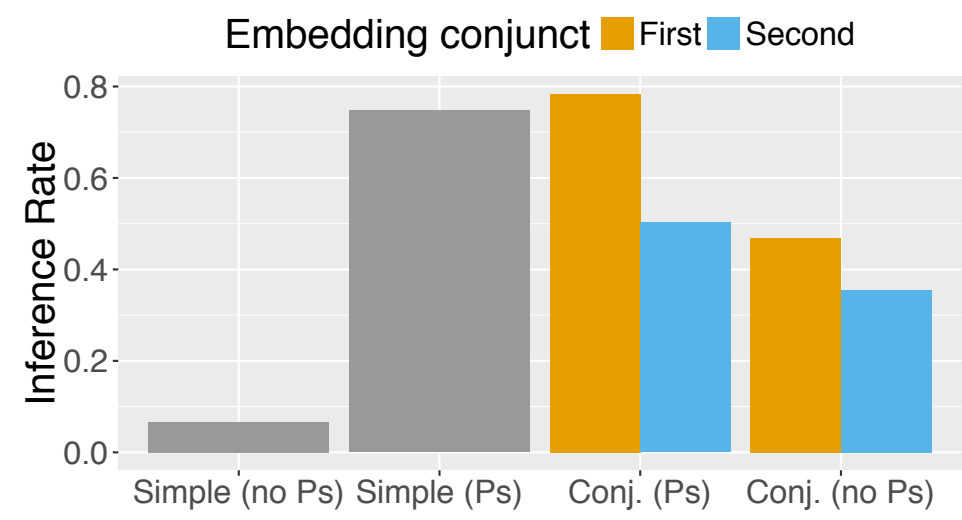

Figure 2 Mean inference rates of $p$ per condition in Experiment 2

for participants and random intercepts for participants and items. They revealed that the contrast between Conj-Ps-First and Simple-Ps was not significant ( $p=0.423)$ but the one between Conj-Ps-Second and Simple-No-Ps was $(p<0.01)$.

\subsection{Discussion}

As in Expt. 1, we found evidence for asymmetry in the form of a significant contrast between Conj-Ps-First and Conj-Ps-Second, replicating our results there in a second population. However, the suspected confound of general order effects, independent of presuppositionality, was also substantiated, given the main effect of order (and corresponding simple effects for both $P s$ and No-Ps). But crucially, we also find a significant interaction between Order and Embedding Type. This indicates that, while there $i s$ an order effect of the kind we hypothesized (perhaps due to parenthetical readings, perhaps due to some other issue), presuppositional variants are subject to an additional, presupposition-specific factor that further enhances the order effect. The most natural explanation of this is an asymmetry in presupposition filtering: a preference for left-to-right filtering over right-to-left filtering.

Once again parallel to the findings of Expt. 1, inference rates were comparable in Conj-Ps-First and Simple-Ps. This further substantiates the conclusion that there is no evidence for right-to-left filtering in the present paradigm, though the new data suggest that this conclusion is also compatible with the hypothesis that there is some right-to-left filtering, together with non-presuppositional inferences having to do with order effects, perhaps due to parenthetical readings. Either way, however, the interaction effect between Order and Embedding Type shows that, even if there is right-to-left filtering, there is significantly less right-to-left than left-to-right filtering.

Furthermore, we find further evidence for the somewhat surprising effect ob- 
served above: even in Conj-Ps-Second, where left-to-right filtering would be expected to be operative, there is an increase in inference endorsements relative to baseline (Simple-No-Ps) and control (Conj-No-Ps-Second) conditions. As before, this could be due to failure of perceiving the relevant entailment relationship, but further work is required to assess this otherwise theoretically surprising finding more carefully.

Finally, another suprising result is that even non-presuppositional variants yield increases in inference endorsement rates, relative to simple (non-conjunctive) nonpresuppositional controls, even when $p^{+}$is in the left conjunct (and thus not susceptible to a parenthetical reading of the kind suggested above). More work is needed to assess in detail what this should be attributed to. Possible explanations could allude to latent presuppositionality of (at least some) of our non-presuppositional predicates, or to the somewhat particular discourse structure induced by the general frame for our stimuli (with one conjunction containing an embedded clause, and the other entailing that embedded clause). It is important to note, however, that the confirmation of presupposition-specific asymmetries through the statistical interaction is independent of such potential other effects.

\section{General discussion}

The central question our experiments aimed to address was whether we find asymmetry in presupposition projection from conjunction, i.e., whether the linear order of a conjunct containing a presupposition trigger and another conjunct that (unidirectionally) entails the relevant presupposition matters for the presupposition of the entire conjunction. We assessed this by further embedding such conjunctions in the antecedent of a conditional, and asking experimental participants whether they would conclude that the presupposition in question holds based on a reliable speaker uttering the sentence. In both of our experiments, we find decreased rates of inference endorsement when the trigger appears in the second conjunct relative to a presuppositional baseline (a comparison which controls for the possibility of local accommodation), suggesting that the material in the first conjunct can filter the presupposition. One worry of the first experiment was that there could be general order effects independent of presuppositionality in this regard. While the second experiment to some extent substantiated such worries, the crucial statistical interaction, with a greater order effect in the presuppositional conditions, reinforces the conclusion that even if there are such independent effects of conjunct order, there are presupposition-specific order effect that can be attributed to asymmetric presupposition projection.

In contrast to what we find for triggers in the second conjunct, inference rates are comparable to those in simple (non-conjunctive) controls when the trigger appeared in the first conjunct, i.e., we find no evidence in our paradigm for right-to-left 
filtering, where later material can prevent the projection of a preceding trigger. This is in line with strictly asymmetric accounts of presupposition projection, and strongly suggests that there is some asymmetry inherent in presupposition projection across conjunction. At the same time, the absence of an effect does not constitute definitive evidence against the possibility of right-to-left filtering, and could be task-specific, or could be explained through the interaction of some filtering together with increased projection due to independent order effects.

A surprising aspect of our results, again present throughout both experiments, was that even in variants where the presupposition was introduced in the second conjunct, inference rates were higher than in simple baseline conditions, suggesting that even left-to-right filtering was not operative as consistently as expected based on standard theoretical accounts. While this could be seen as a challenge to standard assumptions, a more mundane explanation, e.g. in terms of failure to perceive the relevant entailment relation, is also in principle viable. Relatedly, we also find increased inference endorsement rates for non-presuppositional controls in Expt. 2. These, too, require an explanation, but for present purposes, we content ourselves in noting that such explanations should be independent of the presupposition-specific order effects. A general methodological lesson, however, is that these complexities in our results provide a strong motivation for experimental work on projection phenomena, as the various different factors that seem to be at play when global inferences arise based on material introduced in embedded context cannot be teased apart at the level of researchers' own intuitions alone.

\section{Conclusion and further directions}

Projection across conjunction has traditionally been taken to be central evidence that presupposition projection in general is asymmetric. Once we control for independent issues about redundancy, however, the judgments become much less clear, and the recent literature has been split on whether projection across conjunction is asymmetric after all. Using experiments which control for issues of redundancy, as well as distinguishing filtering from local accommodation, we have found evidence that projection across conjunction is indeed asymmetric: we found evidence for left-to-right filtering, but no evidence for right-to-left filtering.

In closing, we will briefly discuss how our results relate to those of a previous study in Chemla \& Schlenker (2012), as well as enumerate a variety of avenues for further research. Chemla and Schlenker's study is the first experimental approach we know of to questions of (a-)symmetry in presupposition projection. They employ both inferential and acceptability experiments to investigate the projection of sentences in which a presupposition trigger appears either sentence-initial or sentence-final, with its presupposition being satisfied by material that preceded or followed it, 
respectively. In particular, their inferential experiments looked at (the corresponding French versions of) sentences like (17) versus (18), containing a presupposition trigger (the corresponding French of too) and material satisfying its presupposition, either preceding or following it. (In addition to disjunctions like (17) and (18), they investigated corresponding conditionals and unless-sentences.)

(17) Ann will not decide to study abroad, or her brother too will make a reasonable decision.

(18) Ann's brother too will make a reasonable decision, or Ann will not decide to study abroad.

Chemla \& Schlenker (2012) measured to what extent participants concluded the inferences in (19) and (20), the rationale being that if the material preceding/following the trigger was considered, participants would only conclude the weak inference in (19) (which contextually corresponds to a conditional presupposition that is relativized to the other disjunct); conversely, if the presupposition of the trigger were simply to project globally, they would conclude the strong inference in (20).

(19) Studying abroad would be reasonable for Ann.

(20) Ann will make a reasonable decision.

In their results, they found that the conditional inference in (19) was preferred in the canonical order, providing evidence that material that precedes the trigger is indeed taken into account, as expected by any theory incorporating a form of left-toright filtering. More surprisingly, they also found that the conditional inference was also endorsed more than the unconditional one in the inverse order, suggesting that material that follows the trigger is also taken into account, thus supporting a theory that also incorporates some form of right-to-left filtering. ${ }^{7}$

The study by Chemla \& Schlenker (2012) was an important first step towards addressing questions about (a-)symmetric effects in projection. There are two noteworthy ways in which our approach goes beyond that of Chemla \& Schlenker (2012). First, Chemla and Schlenker did not look at conjunction, which, again, is taken as the paradigmatic case for asymmetry. Second, they focused on additive particles, to control for presupposition suspension. As Chemla and Schlenker themselves discuss, however, additive particles differ in their properties from other presupposition triggers in important and relevant respects; in particular, these particles' presuppositions project in peculiar ways, as discussed in Soames 1979; Karttunen \& Peters 1979; Chemla \& Schlenker 2012; Romoli 2012; Mandelkern \& Romoli 2017b among

7 Chemla \& Schlenker (2012) did not investigate the potential interaction between type of inference and order, which would be, in our mind, the most convincing measure of asymmetry. 
others. Thus it is not clear how far we can generalize conclusions about presupposition projection in general from projection data on the presuppositions of additive particles.

In sum, while the results in Chemla \& Schlenker 2012 suggest that both left-toright and right-to-left filtering might play a role in presupposition projection, various open questions from their study reinforce the need for more extensive experimental investigation of the question of order effects in projection. ${ }^{8}$ While we did not find any evidence for right-to-left filtering, the results of our study are still consistent with Chemla and Schlenker's claim of the existence of a right-to-left filtering option. Our results advance the debate by showing that, at least when it comes to conjunction, there is a clear asymmetry-subjects are more likely to filter left-to-right than right-to-left - and as yet no evidence for right-to-left filtering.

We turn now to future avenues for research which our study suggests. Our results help clarify the situation regarding projection across conjunction, but they leave open many questions which require substantial follow-up work. A first question is about conjunction in particular. Our results suggest that projection across conjunction is asymmetric as a default, i.e. in the absence of external pressures not to project. But this leaves open the question of whether right-to-left filtering might nonetheless be available as a rescue strategy (as suggested e.g. in Schlenker 2008a). To ascertain whether this is so, future experiments should use similar paradigms to ours (which control for redundancy) while providing context which explicitly leaves open the truth of the presupposition in question. If we find lower projection of $p$ (judged by acceptability ratings) in sentences of the form ' If $S_{p}$ and $p$, then $q$ ' than in controls of the form 'If $S_{p}$, then $q$ ' (to distinguish right-to-left filtering from local accommodation), this would suggest that right-to-left filtering is available when coerced by context, even if not as a default. If by contrast we find no such effect, this would suggest that projection around conjunction is exclusively asymmetric. ${ }^{9}$

A second important question which our results leave open is about the (a)symmetry of other connectives. We cannot assume ex ante that projection across connectives is either uniformly symmetric or uniformly asymmetric, and indeed disjunction in particular has frequently been claimed to exhibit right-to-left filtering, as exhibited by examples like (21) (following Karttunen 1973 a.o.):

(21) Either Mary stopped doing yoga, or she never did yoga.

It's clear that we do not infer from (21) that Mary used to do yoga, a fact which is

8 Schwarz (2015) also investigates (a-)symmetry in projection by using 'again' in antecedent-initial vs. antecedent-final conditional sentences. His results are broadly comparable to those of Chemla \& Schlenker (2012). He also doesn't look at conjunctions, and, again, there is a question as to whether again brings in the same idiosyncratic issues brought in by too.

9 Thanks to Philippe Schlenker for suggesting this follow-up. 
naturally taken as evidence that there is right-to-left filtering across disjunctions. But, as for conjunction, care must be taken here to distinguish filtering from independent pragmatic issues. (21) has the form ${ }^{\ulcorner} S_{p}$ or not $\left.p\right\urcorner$. If $p$ projected from a sentence of this form, we could then conclude $S_{p}$ is true, making the right disjunct redundant. So in cases like this, as has been observed since Gazdar 1979, there is independent pressure to locally accommodate the presupposition. This issue can be avoided, as for conjunctions, by looking instead at sentences of the form ${ }^{\ulcorner} S_{p}$ or not $\left.p^{+}\right\urcorner$, like (22):

(22) Either Mary stopped doing yoga, or she never did Jivamukti yoga.

Here, as for conjunctions, judgments about projection become less clear, and experimental work is needed to assess to what degree subjects will infer that Mary used to do yoga from (22), and to what degree that differs from local accommodation. This will allow us to clarify symmetry properties across different connectives. Finding differences across connectives would pose a striking explanatory challenge, and might suggest that the projection properties of connectives are encoded as a matter of semantic convention (the line suggested by classic work in dynamic semantics like Heim 1983). A uniform picture, by contrast, would be at least prima facie more compatible with approaches which aim to explain projection properties in non-conventional terms, like Schlenker 2008a; Rothschild 2011.

Another important question which our discussion leaves open is the precise nature of the asymmetry which we have found evidence for. Is the asymmetry a matter of linear order, as parsing-based approaches like Schlenker (2008a)'s would suggest, or is it a matter of structure, as classical dynamic approaches would suggest (Chierchia 2009)? This is an important question for understanding where in the linguistic system presuppositions are processed. Conjunctions, at least in languages like English, are unlikely to help us answer this question, as linear and structural asymmetries coincide here (on standard syntactic approaches); recent insight on this question has come from cross-linguistic data (Ingason 2016; Chung 2017), as well as constructions in English with flexible linear order, like conditionals (Mandelkern \& Romoli 2017b,a).

Investigating (a-)symmetry in presupposition projection promises insights into the architecture of one characteristic component of natural language. In addition, it may inform theories of redundancy (which include both symmetric and asymmetric versions, as noted above), anaphora, and epistemic modals (which are argued in Mandelkern 2017 to be interpreted relative to an exclusively symmetric system of local contexts). The study of these latter phenomena, likewise, may indirectly help determine the best theory of presupposition projection. Many more new questions are raised here than answered, but we hope that our experimental results, as well as the paradigm we developed, advance our understanding of these subtle issues. 
Mandelkern, Zehr, Romoli, \& Schwarz

\section{References}

Chemla, Emmanuel. 2008. Similarity: Towards a unified account of scalar implicatures, free choice permission and presupposition projection. Manuscript, Ecole Normale Supérieure, Paris and MIT. http://www.emmanuel.chemla.free. fr/Material/Chemla-SIandPres.pdf.

Chemla, Emmanuel \& Philippe Schlenker. 2012. Incremental vs. symmetric accounts of presupposition projection: an experimental approach. Natural Language Semantics 20(2). 177-226. doi:10.1007/s11050-012-9080-7.

Chierchia, Gennaro. 2009. On the explanatory power of dynamic semantics. Handout from talk at Sinn und Bedeutung 14.

Chung, WooJin. 2017. An evaluation-sensitive model for local context computation. Unpublished manuscript NYU.

Fox, Danny. 2008. Two short notes on Schlenker's theory of presupposition projection. Theoretical Linguistics 34(3). 237-252.

Gazdar, Gerald. 1979. Pragmatics: Implicature, Presupposition and Logical Form. New York: Academic Press.

George, Benjamin Ross. 2008. Presupposition Repairs: A Static, Trivalent Approach to Predicting Projection. University of California, Los Angeles MA thesis. http://www.semanticsarchive.net/Archive/2ZiYmEyN/brgeorge_ma.pdf.

Heim, Irene. 1983. On the projection problem for presuppositions. In Michael Barlow, Daniel P. Flickinger \& Nancy Wiegand (eds.), The Second West Coast Conference on Formal Linguistics (WCCFL) 2, 114-125. Stanford Stanford University Press. doi:10.1002/9780470758335.ch10.

Ingason, A.K. 2016. Context updates are hierarchical. Glossa 1(1). 1-9.

Karttunen, Lauri. 1973. Presuppositions of compound sentences. Linguistic Inquiry 4(2). 167-193.

Karttunen, Lauri. 1974. Presupposition and linguistic context. Theoretical Linguistics 1(1-3). 181-194. doi:10.1515/thli.1974.1.1-3.181.

Karttunen, Lauri \& Stanley Peters. 1979. Conventional implicature. Syntax and Semantics 11. 1-56.

Katzir, Roni \& Raj Singh. 2013. Hurford disjunctions: embedded exhaustification and structural economy. In Urtzi Etxeberria, Anamaria Fǎlăuş, Aritz Irurtzun \& Bryan Leferman (eds.), Sinn und Bedeutung 18, .

Mandelkern, Matt \& Jacopo Romoli. 2017a. Parsing and presupposition in the calculation of local context. Semantics \& Pragmatics In press.

Mandelkern, Matthew. 2017. Coordination in conversation: Massachusetts Institute of Technology PhD dissertation.

Mandelkern, Matthew \& Jacopo Romoli. 2017b. Parsing and presuppositions in the calculation of local contexts. Semantics and Pragmatics 10(17). 
doi:10.3765/sp.10.7.

Mayr, Clemens \& Jacopo Romoli. 2016. A puzzle for theories of redundancy: Exhaustification, incrementality, and the notion of local context. Semantics and Pragmatics 9(7). 1-48. doi:10.3765/sp.9.7.

Romoli, Jacopo. 2012. A solution (or two) to Soames' problem: Presuppositions, conditionals and exhaustification. International Review of Pragmatics 4(2). 153-184.

Romoli, Jacopo \& Matthew Mandelkern. 2016. Hierarchical structure and local contexts. To appear in Sinn und Bedeutung 21.

Rothschild, Daniel. 2008. Presupposition projection and logical equivalence. Philosophical Perspectives, 22, Philosophy of Language 473-497.

Rothschild, Daniel. 2011. Expressing credences. In Proceedings of the Aristotelian Society 112 1, 99-114.

Schlenker, Philippe. 2008a. Be articulate: a pragmatic theory of presupposition projection. Theoretical Linguistics 34(3). 157-212. doi:10.1515/THLI.2008.013.

Schlenker, Philippe. 2008b. Presupposition projection: Explanatory strategies. Theoretical Linguistics 38(3). 287-316. doi:10.1515/THLI.2008.021.

Schlenker, Philippe. 2009. Local contexts. Semantics and Pragmatics 2(3). 1-78. doi:10.3765/sp.2.3.

Schwarz, Florian. 2015. Symmetry and incrementality in conditionals. In Florian Schwarz (ed.), Experimental Perspectives on Presuppositions 45 Studies in Theoretical Psycholinguistics, 195-213. Springer.

Soames, Scott. 1979. A projection problem for speaker presuppositions. Linguistic Inquiry 10(4). 623-666.

Stalnaker, Robert. 1974. Pragmatic presuppositions. In Milton K. Munitz \& Peter Unger (eds.), Semantics and Philosophy, 197-213. New York: New York University Press.

Stalnaker, Robert. 1978. Assertion. In Peter Cole (ed.), Syntax and semantics 9, 315-322. New York: Academic Press.

Matthew Mandelkern

All Souls College

University of Oxford

OX1 4AL

United Kingdom

matthew.mandelkern@gmail.com
Jérémy Zehr

University of Pennsylvania

3401-C Walnut, Suite 300

Philadelphia, PA

United States

jeremy.e.zehr@gmail.com 
Mandelkern, Zehr, Romoli, \& Schwarz

Jacopo Romoli

Ulster University

Shore Road, Newtownabbey

BT37 0QB

United Kingdom

j.romoli@ulster.ac.uk
Florian Schwarz

University of Pennsylvania

3401-C Walnut, Suite 300

Philadelphia, PA

United States

florians@ling.upenn.edu 